\title{
Introduction from the new Editors-in-Chief
}

\author{
Steven Swift $^{1} \cdot$ Rebecca Rogers ${ }^{2}$
}

Received: 2 November 2016 / Accepted: 2 November 2016 / Published online: 21 November 2016

(C) The International Urogynecological Association 2016

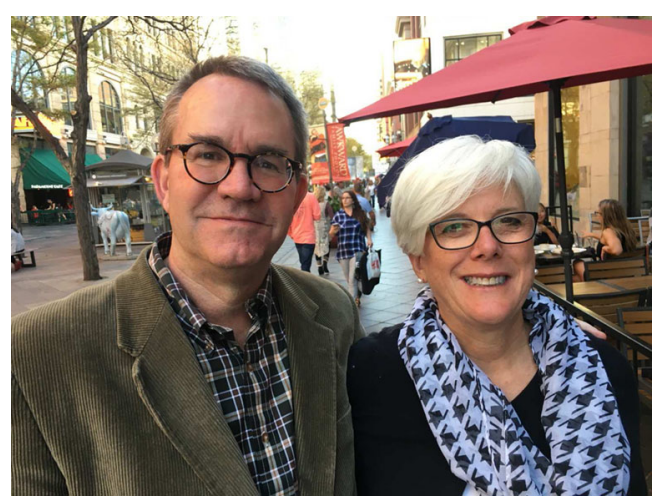

As we begin our tenure as Editors-in-Chief of the International Urogynecology Journal (IUJ), we would like to thank the previous Editors-in-Chief for establishing the IUJ as the premier journal in the field of urogynecology. Our journal is the "go-to" journal for practicing urogynecologists across the globe, and the IUJ publishes more urogynecology articles than any other subspecialty journal in the field. We fully recognize that the greatest strength of the $I U J$ is the international makeup of our Editors, readership, reviewers, and authors. Our main task

Steven Swift

swifts@musc.edu

Rebecca Rogers

RRogers@salud.unm.edu

1 Medical University of South Carolina, Charleston, SC 29425, USA

2 University of New Mexico, Albuquerque, NM 87131, USA as incoming Editors-in-Chief will be to maintain that representation and promote further engagement with scientists and clinicians internationally so that our journal continues to attract and publish the best manuscripts in the field of urogynecology.

We feel strongly that our mission is to provide a platform for the publication of the best that we as urogynecologists have to offer. This means that we will reach out to our readership, Editorial Board and fellow Editors to find new and innovative ways to ensure that the best science is published with efficient, high-quality reviews that are completed in a timely manner. To achieve this, we will focus on improving the work flow of the Editors such that their job in managing submissions is efficient, effective, and timely. This will mean further reductions in time-to-decision and more productive manuscript reviews for authors. We will focus on providing better guidance to our reviewers so that we receive only high-quality reviews from all who participate in the manuscript review process. Finally, we will focus on making the manuscript submission process an easy one that provides authors with a simple process for submission and a short time to decision. Although these processes have already improved substantially, our job is to determine how to improve them even further.

As times change, we as Editors-in-Chief will work to put the IUJ in a position to flourish. This will means engaging new international communities that have up and coming research units. We must also find new and different ways to engage younger readers while maintaining our standing amongst our traditional readership. We look forward to working with the International Urogynecological Association leadership to promote research and publication in our field and work closely with our partners at Springer to promote the journal. 
We look forward to serving the readership of the IUJ as the new Editors-in-Chief. We hope that after our tenure is complete we will have done as much to foster better science and to promote urogynecology as our predecessors, Drs Dwyer and Riss, have done. To be successful, we know that it will take input from the many people who already make significant contributions to the IUJ. We would like the readership, re- viewers, and Editors to know that we are always willing to discuss new ideas and will keep an open mind when it comes to promoting the journal. We look forward to the productive years ahead.

Sincerely,

S Swift, R Rogers 